\title{
Ricoeur y la teleología implícita en la obra freudiana
}

Ricoeur and the implicit teleology in Freud's work

Dra. Ana Escríbar anita.escribarwicks@gmail.com

Profesora Emérita

Universidad de Chile

Chile

\section{Resumen}

Se analiza la crítica que Ricoeur hace desde la perspectiva filosófica a lo que considera la unilateralidad arqueológica de la interpretación freudiana de la cultura; se revisa su sugerencia de una complementación teleológica a la arqueología psicoanalítica que -apoyada en la fenomenología del espíritu hegeliana- redunde en un enriquecimiento de la nueva comprensión freudiana del sí mismo y de la cultura y tienda a la unificación del lenguaje dividido por el "conflicto de las interpretaciones". Se destaca cómo el debate con Freud se mantiene permanentemente en el trasfondo del pensamiento ricoeuriano, tanto en su estudio del conflicto de las hermenéuticas, como en su teoría del símbolo y del texto, en su comprensión narrativa del "sí mismo" y de la cultura, y en su fundamentación última de la ética en el deseo de ser y en el esfuerzo por existir.

Palabras clave: arqueología, teleología, fenomenología, hermenéutica, símbolo.

\begin{abstract}
The paper analyses Ricoeur's criticism from a philosophical perspective of what he considers the arquaeological unilaterality of the Freudian interpretation of culture; it examines his suggestion of a teleological complementation to the psicoanalitical arqueology -based on the hegelian phenomenology of the spirit- that might lead to an enrichment of Freud's new understanding of self and culture and to the unification of language, divided by the "conflict of interpretations". It emphasizes the permanence of the debate with Freud in the background of Ricoeur's thinking, just as much in his study of the hermeneutic's conflict as in his theory of symbol, in his narrative


understanding of self and culture, and in his last foundation of ethics in the desire of being and in the effort to exist.

Key words: arquaeology, teleology, phenomenology, hermeneutic, symbol.

\section{Introducción}

La lectura que Ricoeur (1970) hace de la interpretación freudiana de la cultura no representa un capítulo aislado en el pensamiento del filósofo francés. En realidad, podría decirse que en toda su obra posterior a 1965, fecha en que se publica el original francés del libro mencionado, el debate con Freud -del cual Ricoeur dice que representa lo esencial de su lectura de la interpretación freudiana de la cultura- se mantiene permanentemente en el trasfondo -cuando no explícitamentetanto en su estudio del conflicto de las hermenéuticas, como en su teoría del símbolo y del texto, en su comprensión narrativa del "sí mismo", y en su fundamentación última de la ética en el deseo de ser y en el esfuerzo por existir.

Ricoeur pone mucho énfasis en aclarar de partida los términos de este debate. Reconoce, en primer lugar, que -no siendo psicoanalista y no habiéndose sometido jamás al análisis- sus observaciones y comentarios referentes a la obra freudiana no se hacen desde la perspectiva de la psicología, sino desde la disciplina filosófica.

En consecuencia -afirma- su crítica no se refiere a los posibles efectos terapéuticos del método psicoanalítico, sino a la ausencia de explicación para el logro de ciertos momentos en el desarrollo de la vida psíquica y de la cultura, cuya presencia Freud expresamente reconoce. Se trata de la "identificación" y la "sublimación", relacionadas ambas con una aparente superación del complejo edípico que carece de fundamento dentro de la teoría freudiana, a pesar de que están supuestos en el acceso a la vida adulta y en la creación artística, respectivamente.

Freud entiende por identificación el proceso mediante el cual el individuo reemplaza el deseo de dar muerte al padre para sustituirlo en el amor de la madre, por el reconocimiento de aquel como modelo que permitirá al hijo reproducir -con otra mujer que para él la sucederá- la relación entre 
el padre y la madre. La sublimación la define como la desviación de la energía propia del deseo edípico hacia otro objeto ajeno a esa relación, con lo que el deseo pierde su carácter narcisista y así liberado- se hace creador.

Desde la perspectiva freudiana, el arte en todas sus formas es el único lugar dentro de la cultura en el que es posible la auténtica creación; vale decir, donde -a diferencia de lo que según Freud sucede en la religión y en la ética- lo producido no es mera repetición disfrazada del conflicto edípico, sino que lo trasciende al esbozar posibles soluciones. Freud ejemplifica dicha sublimación con su análisis de la Gioconda cuya sonrisa empieza remitiendo a la de la madre de Leonardo, para luego dejarla atrás y mostrar en esos mismos labios otros objetos posibles.

El debate que Ricoeur mantiene con Freud a lo largo de su obra no pretende negar el gran aporte freudiano a la comprensión de sí mismo y de la cultura, que el filósofo francés reconoce con entusiasmo; intenta, en cambio, mostrar que la plena comprensión y aprovechamiento de ese aporte exige la explicitación de elementos que están allí implícitos y cuyo desarrollo permitiría corregir esos vacíos que se mencionan en los párrafos anteriores.

Ricoeur se refiere, fundamentalmente, a la complementación de la arqueología propia de la explicación freudiana y de toda la Escuela de la Sospecha, con la teleología de la conciencia, tal como es desarrollada por la fenomenología del espíritu hegeliana, pero despojada de la totalización prematura del "saber absoluto" en la que aquella desemboca; sin embargo -afirma el filósofo francés- no se trata de imponer desde fuera algo ajeno a la comprensión que Freud presenta del hombre y de la cultura, sino de permitir que aflore la aspiración que efectivamente está entremezclada con la determinación causal en distintas proporciones, según el nivel de la vida psíquica del que se trate.

Considera Ricoeur que la complementación mencionada permitiría la unificación del lenguaje que -a pesar de centrar la atención de la reflexión filosófica contemporánea con su "giro lingüístico"- se encuentra, no obstante, quebrado; dividido por el "conflicto de las interpretaciones" entre quienes quieren destruir los símbolos y los mitos para que el hombre se atreva a pensar y quienes quieren destruir los ídolos, para recuperar el valor de esos símbolos y mitos como reveladores de la experiencia que el hombre tiene de sí mismo y de su relación con 
"lo sagrado". Se trata en el fondo del enfrentamiento de quienes consideran necesario romper con las tradiciones para que el hombre entre en la mayoría de edad y de quienes -por el contrario- reconociendo que ellas nos constituyen, incluso en nuestras posibilidades de rebeldía, consideran necesario interpretarlas y re-interpretarlas cada vez, para desplegar sus diferentes e inagotables posibilidades de sentido.

Ricoeur tiene plena conciencia de la enormidad de la tarea implicada en esa aspiración y declara expresamente que no pretende ni siquiera bosquejar esa gran filosofía del lenguaje que pudiera llegar a unificarlo; se limita -dice- a apuntar en esa dirección.

El presente trabajo intentará esbozar este apuntar unitario ricoeuriano, para mostrar sus posibles efectos sobre la comprensión de sí mismo y de la cultura.

\section{Interpretación de la simbólica del mal.}

Ricoeur, mediante la hermenéutica de la simbólica del mal (1969), pone al descubierto ciertos rasgos de la comprensión del mal previos a la elaboración teológico-filosófica, tal como aparecen en los Libros Sagrados judeo-cristianos; estos rasgos los extenderá a todos los símbolos dentro de esa tradición; esto es, a todos los "signos de grado compuesto donde el sentido, no conforme con designar una cosa, designa otro sentido que no podría alcanzarse, sino en y a través de su enfoque o intención" (Ricoeur, 1970, p. 18).

Ahora bien, debido a la omnipotencia e infinita bondad que aparecen como atributos divinos dentro de esa tradición, el problema del origen del mal en un mundo creado por Dios a partir de la nada, detenta un lugar destacado dentro de la reflexión teológico-filosófica occidental, surgiendo así todas las teodiceas que intentan explicarlo.

La tradición greco-judeo-cristiana, en relación con dicho problema, ha dado lugar a dos comprensiones contrapuestas del mal; por un lado, la trágica, que lo concibe como algo exterior al hombre, de lo que éste no es responsable puesto que lo asalta desde fuera como un contagio, o se le impone como un destino. Por otro, la comprensión ética que lo interioriza, culpando a la libertad humana de una falta o pecado que provoca la entrada en el mundo del mal físico como castigo por ello. 
Esta dualidad en la comprensión de dicho problema, es la que impulsa a Ricoeur (1969) a emprender la interpretación de la simbólica del mal centrando su atención en textos religiosos referentes a la "confesión de los pecados", pertenecientes a la tradición judeo-cristiana. Descubre allí tres etapas que van desde la percepción del mal "como" una mancha, a la del mal "como" la esclavitud del pueblo elegido generada a partir de la ruptura de la relación con Dios, hasta la de su comprensión "como" una carga o un peso, de quienes no responden ya ante Dios, sino ante la propia conciencia.

La hermenéutica ricoeuriana nos muestra, pues, una evolución de la simbólica del mal que va desde una comprensión trágica a una comprensión ética, desde la exterioridad del mal en relación con el hombre hasta una interioridad creciente que en el pueblo elegido se expresa en una responsabilidad colectiva ante Dios, para llegar luego a presentarse como responsabilidad personal.

Esta comprensión ética del mal culmina en el Antiguo Testamento con la narración del Génesis en la que Adán y Eva -al desobedecer el mandato divino- aparecen como responsables de la entrada del mal en el mundo. Sin embargo, en esta culminación mítica de la comprensión ética aparece -con la serpiente que tienta- un mal previo, exterior, del que no es responsable la libertad humana y que Ricoeur designa como "mal ya allí".

A partir de esta descripción de la evolución de la comprensión del mal, Ricoeur concluye que ni la exterioridad del mal en la comprensión trágica, ni su interioridad en la comprensión ética, vale decir, ni la irresponsabilidad o la responsabilidad humanas en relación con el mal, son jamás completas; lo que hay es una combinación de ambas que en un desarrollo dialéctico avanza desde el predominio de la exterioridad al de la interioridad, sin que en ningún momento la una o la otra aparezcan en estado puro.

Esta permanencia de lo trágico, de la exterioridad, en el mito adánico -único antropocéntrico en referencia a dicho problema- podría considerarse como un fracaso del intento de explicar el origen del mal mediante el mito, lo que incita a la reflexión filosófica. Los dos pensadores más destacados en la dirección de una comprensión ética del mal son San Agustín y Kant, pero el intento de ambos se frustra en la medida en que culmina con la aparición de un mal "ya allí" que 
la contradice; la noción de "pecado original" en el primero, que reúne en un concepto imposible o pseudo concepto -que es más bien un "símbolo racional"- la libertad de la voluntad y la culpa por el pecado de Adán y Eva heredada por todos los hombres; la de "siervo-arbitrio" en el segundo, que -ante la inexplicable presencia conjunta de una tendencia al bien y una inclinación al mal en el ser humano- reconoce el problema del origen del mal como un misterio insoluble para la razón. (Ricoeur, 1969).

\section{El conflicto de las Interpretaciones; teoría del símbolo y del texto.}

Ricoeur (1970) se refiere al papel central del lenguaje en la reflexión filosófica contemporánea, enumerando varios de los filósofos que ejemplifican esta situación, Wittgenstein, Husserl. Heidegger, incluyendo a Freud y al psicoanálisis. Este último participa en el actual debate contemporáneo sobre el lenguaje, en primer lugar, por su interpretación de los sueños, ya que dicha interpretación no se dirige directamente a lo soñado, sino a la narración del soñador a partir de la cual el analista intenta reemplazar el relato original por otro, que refleje el deseo con mayor fidelidad.

Porque en el sueño - como efecto de la censura- el deseo se presenta disfrazado y el relato que lo narra se formula en un lenguaje distorsionado y dotado de un doble sentido o de sentidos múltiples.

Ahora bien, Freud presenta todos los productos psíquicos, desde la locura hasta las creaciones culturales, como análogos al sueño; vale decir, como lenguaje distorsionado, enraizado en el deseo que -al igual que el sueño- quiere decir algo distinto de lo que dice. Al respecto afirma Ricoeur: "El sueño y sus análogos se inscriben así en una región del lenguaje que se anuncia como lugar de significaciones complejas donde otro sentido se da y se oculta en un sentido inmediato; llamamos símbolo a esa región del doble sentido" $(1970,10)$. Y es este carácter ambiguo del lenguaje en el que la cultura se expresa, el que exige la interpretación o hermenéutica, como "interpretación del doble sentido" $(1970,11)$

Pero la preocupación por los símbolos no es exclusiva del psicoanálisis; por lo pronto, es compartida por los otros dos grandes maestros de la Escuela de la Sospecha, Nietzsche y Marx, 
que además comparten con Freud la hazaña de haber descentrado la fuente del sentido, llevándola fuera de la conciencia. Centra también la atención de la Fenomenología de la Religión; esta última, sin embargo, no concibe la interpretación como un alzamiento de las máscaras que -a la vez- muestran y disimulan el deseo inhibido, la voluntad de poder o el conflicto social, sino como manifestación de lo sagrado que en los símbolos aflora y también se oculta.

Ante este enfrentamiento de las interpretaciones, Ricoeur $(1970,11)$ plantea a Freud una primera pregunta que en realidad las encierra a todas: "¡el mostrar ocultar del doble sentido es siempre disimulación de lo que quiere decir el deseo, o bien puede ser a veces manifestación, revelación de lo sagrado?"

Este enfrentamiento entre las dos formas de entender el sentido de los símbolos es lo que Ricoeur denomina "conflicto de las interpretaciones"; en primer lugar está la interpretación de la Escuela de la Sospecha - ella misma dividida, porque Nietzshe, Freud y Marx- como vimos en el párrafo anterior, sitúan en diversos objetos la fuente del sentido y, consecuentemente, diseñan diferentes métodos para desenmascararlo (genealogía, psicoanálisis y crítica de las ideologías), pero coinciden en la explicación arqueológica del sentido de los símbolos. En segundo lugar, la de la Fenomenología de la Religión representada, entre otros, por Mircea Eliade.

Ahora bien, Ricoeur considera que esta contraposición no es arbitraria, sino que se origina a partir de una característica que define la naturaleza misma del símbolo y que él desprende de la interpretación de la simbólica del mal. (Ricoeur, 1969). Surge a partir del doble apuntar del símbolo en dirección a su origen en el pasado, en primer lugar, con lo que apunta hacia un determinismo; y en dirección al futuro -en segundo lugar- a partir de una interpelación que el hombre recibe, interpreta y reinterpreta una y otra vez a lo largo de la historia.

Estas dos direcciones -nos dice el filósofo francés- aparecen entretejidas en los mismos símbolos, siendo constitutivas de su "sobre-determinación"; de manera que nuestros más elevados ideales están ya germinalmente presentes en el origen en apariencia enteramente determinado causalmente de nuestros símbolos. 
Posteriormente, Ricoeur (1984) preferirá como punto de partida de su hermenéutica a la "metáfora", a la que define como "núcleo semántico" del símbolo; vale decir, como lo que lleva a la palabra aquella parte de este último que puede ser dicha. La metáfora pertenece, pues, enteramente al ámbito del lenguaje, mientras el símbolo remite siempre a algo que lo trasciende "lo que hay que decir", esgrimido por el poeta, los fantasmas de lo onírico para el psicoanálisis, o los símbolos cósmicos en la fenomenología de la religión- impulsando, así, permanentemente la interpretación metafórica.

La metáfora supone una frase que establece entre sus componentes una relación de proximidad que rompe con su sentido literal; por ejemplo, "la naturaleza es un templo cuyos pilares vivientes"....

Esa proximidad -afirma Ricoeur- es inaugurada por la metáfora, más que descubierta por ella; en consecuencia, puede afirmarse que ese breve "texto" que ella es opera una reordenación de la realidad, abriendo así un nuevo mundo posible.

Por texto entiende Ricoeur las huellas que el hombre deja de sí mismo en el arte (pintura, escultura, literatura, música, etc.) y en la religión, en la moral, en la acción significativa; esto es, en todo aquello que trasciende las intenciones del autor, los determinantes que explican su origen, el público al que originalmente estaba dirigido, superando la transitoriedad generada por todas esas amarras y quedando abierto a las interpretaciones sucesivas de las diferentes generaciones.

El texto es, pues, una "obra", algo que adquiere autonomía, una forma de "objetividad" que habiéndose liberado de sus determinaciones de origen- abre un nuevo mundo posible en el que el hombre puede re-interpretarse a sí mismo en función de nuevos horizontes.

La proximidad entre teoría del texto a partir de la metáfora y teoría del símbolo, por otra parte, se pone de manifiesto con la reaparición en este nuevo ámbito del conflicto de las interpretaciones. Nuevamente las lecturas se dividen y se considera al texto arqueológicamente determinado a partir de las condiciones de su origen, o se lo entiende íntegramente en función de la aspiración. 


\section{Arqueología y teleología en la obra de Freud.}

Sigamos ahora a Ricoeur cuando nos dice:

Volvamos a Freud. Decíamos que el psicoanálisis es un análisis y no cabe completarlo con una síntesis. Eso no admite réplica. Lo que creo poder mostrar es que este análisis no puede ser comprendido, en su estructura 'regresiva' más específica, a no ser en contraste con una teleología de la conciencia que no le sea exterior sino algo a lo que aquél nos remite por sí mismo (1970, 414).

En el párrafo citado - que podría ser considerado como la hipótesis de trabajo que guía su lectura de Freud- el filósofo francés reconoce y valora el carácter analítico de la descripción que Freud hace de los distintos elementos implicados en la conciencia de sí y en sus productos, tales como los sueños, la religión, la moral y el arte; reconoce que da de ellos una explicación arqueológica, atribuyendo a todos un mismo origen, el deseo, cuya inhibición libera la energía que alimenta dichos productos, equivalentes entre sí, en la medida en que expresan ese deseo en distintos ámbitos, disfrazándolo y dándole una satisfacción sustitutiva.

Ricoeur reconoce igualmente allí que no sería legítimo pretender completar el análisis freudiano imponiéndole desde fuera una síntesis que -reemplazando la dirección "regresiva" de aquélintentara relacionar los distintos componentes de la conciencia de sí dando razón de cada uno de ellos con el resultado de su unión en un momento ulterior de su evolución, como lo hace la Fenomenología del Espíritu hegeliana.

Al hablar de síntesis, Ricoeur hace referencia explícita a dicha fenomenología y a su descripción del proceso conducente a la aparición de la conciencia de sí; esta última -nos dice Hegel- asiste en primera instancia a la manifestación del mundo sin saber de sí misma, ni de su propia presencia en medio de esa manifestación; pero un desarrollo de carácter dialéctico la conduce hasta desembocar en el "saber absoluto" en el que llega a conocerse enteramente a sí misma, con todos los rasgos alcanzados a través de su evolución. Desarrollo dialéctico, porque avanza a lo largo de las distintas etapas evolutivas atravesando -cada vez- tres momentos (afirmación o tesis, 
negación o antítesis y negación de la negación o síntesis), en una espiral que implica cada vez un aparente retorno al punto de partida, pero a otro nivel.

Lo ejemplificaremos, simplificándolo, con la relación "amo-esclavo", que designa una de las figuras más conocidas del desarrollo dialéctico hegeliano. Primero, la conciencia se enfrenta a otra conciencia y -exigiendo reconocimiento mutuo- entablan entre ellas una lucha a muerte; pero una de las conciencias siente temor a la muerte y acepta el dominio de la otra, que -a cambio- le perdona la vida; surgen así "amo" y "esclavo"; segundo, el amo -que ha sido reconocido- entrega la relación con las cosas al esclavo, limitándose él a su goce; tercero, la relación de trabajo con las cosas que exige la postergación del goce, forma al esclavo y el amo -que ahora depende de él para satisfacer sus necesidades- debe, a su vez, reconocerlo.

Entonces y solo entonces -gracias al mutuo reconocimiento- surge la conciencia de sí que permite comprender los dos momentos anteriores que -explicados únicamente a partir del enfrentamiento de las conciencias- entregaría una visión unilateral y, consecuentemente, reductora de un proceso que solo se comprende integralmente en su sentido a partir del reconocimiento mutuo en el que culmina.

Lo que Ricoeur afirma en el trozo antes citado, es que no podría añadirse desde fuera una síntesis; pero además no hace falta hacerlo, porque la teleología -sin haber sido desarrollada por Freud- está, sin embargo, implícita en su pensamiento; y lo está, porque le regresión es inconcebible sin una progresión, la arqueología solo se hace inteligible mediante el contraste con una teleología. Vale decir, el análisis solo es practicable si hay una síntesis que lo posibilite, ya que el primero supone distinguir, separar los elementos que en la última están integrados. Lo está -concretamente- porque si bien la arqueología explica el origen de algo, no basta para comprender el desarrollo de ese algo que implica la aparición de elementos no contenidos en el origen.

Así, si la arqueología freudiana con su explicación determinista nos muestra al hombre como preso de su infancia, es insuficiente para explicar cómo logra él liberarse de esa prisión, cómo llega a ser adulto y cómo con los productos de su cultura -por lo menos con el arte, que no se 
limita a la repetición del conflicto edípico, sino que esboza posibles soluciones- se convierte en creador.

Pero, y en esto reside específicamente el aporte de Ricoeur a la interpretación de la cultura, la teleología tampoco es íntegramente posible sin la arqueología. Es interesante y aplicable a nuestro problema lo que Kant dice para caracterizar las construcciones racionales que no se apoyan en la experiencia, que carecen de base empírica. Kant compara la razón en esas circunstancias a una paloma a la que se la hiciera volar en el vacío; en primera instancia el ave sentiría -llena de gozo- que sus alas no encuentran resistencia, ya que no hay aire que se les oponga; pero muy pronto constataría que -sin esa resistencia- sus alas no pueden sustentarla e inevitablemente se precipitaría hasta estrellarse contra el suelo. Así, la razón -cuando emprende el vuelo sin apoyo empírico- construye con toda facilidad hermosos edificios que, sin embargo, conducen finalmente a la contradicción de la razón consigo misma, como sucede -dice Kant- en la metafísica, cuando la razón intenta probar el libre albedrío, la vida eterna y la existencia de Dios.

Algo similar acontece cuando la teleología pretende dar cuenta por sí sola del surgimiento de la conciencia de sí y de sus productos; sin el reconocimiento de la presencia de la arqueología, ella se convierte en disfraz, en disimulo, en ilusión; cae - a menudo- en totalizaciones prematuras, esto es, en la convicción de haber alcanzado la meta última del desarrollo evolutivo -como le sucede a Hegel con el "saber absoluto"- en circunstancias que el sentido investigado por la hermenéutica jamás culmina.

Este doble apuntar arqueológico y teleológico de los símbolos permite entender el surgimiento del conflicto de las hermenéuticas; ambas están legitimadas por su interpretación de una de las direcciones del apuntar de los símbolos o de los textos; pero también ambas son reduccionistas, en la medida en que desconocen uno de los elementos constitutivos de lo que pretenden interpretar. Al respecto es interesante recordar que esas direcciones contrapuestas del apuntar se dan entrelazadas en los mismos símbolos; de manera que el desarrollo del espíritu se da inevitablemente integrado con el lenguaje distorsionado en el que se expresa el deseo. 


\section{Conclusiones}

Ricoeur considera que a partir de la Escuela de la Sospecha y su descentramiento de la fuente del sentido situada por ella fuera de la conciencia, la filosofía reflexiva -tendiente a la comprensión del sí mismo- es necesariamente hermenéutica; vale decir, no existe una intuición de sí mismo que posibilite el auto-conocimiento directo; este último exige un "camino largo", un rodeo por las obras para que mediante su interpretación el hombre alcance la auto-comprensión.

El filósofo francés, declara expresamente que estudia a Freud en busca de una profundización de la comprensión de sí mismo y de la cultura. Pero llega a la conclusión de que la explicación unilateralmente arqueológica que la obra freudiana hace al equiparar la cultura -salvo en lo que respecta al arte- con los sueños, como expresión disfrazada del deseo inhibido, obstaculiza esa profundización; porque si bien esas obras se esculpen a partir de los fantasmas de nuestros sueños, esos mismos fantasmas contienen ya en germen los ideales que -con la mediación del trabajo- abrirán el camino hacia nuevos mundos posibles en los que el hombre pueda reinterpretarse una y otra vez.

Al respecto, plantea fundamentalmente dos interrogantes; si el hombre es el único animal que permanece preso de su infancia ¿cómo logra su madurez? Y si no puede lograrla, ¿cómo se explica la creación artística en la que Freud reconoce -a diferencia de lo que sucede con la religión y la moral- una auténtica superación de esa condición infantil, expresada en una obra que no se limita a la repetición del conflicto edípico, sino que lo sublima? Ricoeur sostiene que esas interrogantes exigen el reconocimiento de una teleología implícita junto a la arqueología explícita en toda la obra freudiana. Vale decir, la presencia -junto al deseo narcisista de poseer y entretejido con él- de un deseo de ser que exige el reconocimiento de sí mismo y del otro como iguales.

Ricoeur aboga, entonces, por la integración de ambas hermenéuticas, la de la Escuela de la Sospecha que pretende explicar arqueológicamente el surgimiento de la cultura, y la de la Fenomenología del Espíritu que pretende describirlo retroactivamente, desde un fin a lograr. 
Aspira, pues, a superar el "Conflicto de las Hermenéuticas" para alcanzar la unidad del hablar, perdida simbólicamente en la construcción de la Torre de Babel, con la que los hombres -tal como Adán y Eva en el Jardín del Edén- confiaban llegar a ser como dioses, esperanza que quizás hoy se ha visto renovada con el nuevo poderío técnico.

Ricoeur descubre la posibilidad y la necesidad de una complementación entre ambas hermenéuticas que traiga consigo una corrección mutua; que libere a una de su unilateralidad arqueológica que le impide dar cuenta de la sublimación, y a la otra le evite el olvido del carácter parcial de toda interpretación que hace de ella una tarea interminable, evitando así las totalizaciones, siempre trágicamente prematuras.

Lo anterior obliga a Ricoeur a reconocer que su propia propuesta unificadora no puede aspirar a una realización definitiva, que debe limitarse a señalar un camino en esa dirección inalcanzable. Esto, que otorga coherencia a su pensamiento, no le resta importancia ni validez, sino que reafirma el rasgo más profundo de su fenomenología-hermenéutica: el reconocimiento del carácter inagotable del sentido.

\section{Bibliografía}

Freud, S. (1968). El porvenir de una ilusión. En Obras Completas Tomo II. Editorial Biblioteca Nueva: Madrid.

Freud, S. (1968). Totem y tabú. En Obras Completas Tomo II. Editorial Biblioteca Nueva: Madrid.

Freud, S. (1968). El malestar de la cultura. En Obras Completas. Tomo III. Editorial Biblioteca Nueva: Madrid 1968.

Freud, S. (1969). Moisés y la religión monoteísta. En Obras Completas Tomo III. Editorial Biblioteca Nueva: Madrid.

Kant, I. (1973). Critique of pure reason. The Macmillan Press LTD: London 
Kant, I. (1981). La religión dentro de los límites de la mera razón. Alianza Editorial: Madrid.

Hegel G. (1939). La phénoménologie de l'esprit. Aubier, Éditions Mantaine: Paris. Tomo I

Ricoeur, P. (1969). Le conflit des interpretations. Essais d'herméneutique. Editions du Seuil: Paris.

Ricoeur, P. (1970). Freud: una interpretación de la cultura. Siglo XXI editores: México.

Ricoeur, P. (1984). Educación y política. Editorial Docencia: Buenos Aires.

Ricoeur, P. (1985). Hermenéutica y acción. Editorial Docencia: Buenos Aires.

Ricoeur, P. (1996). Sí mismo como otro. Siglo XXI Editores: España.

Ricoeur, P. (2001). Del Texto a la Acción. Ensayos de hermenéutica II. Fondo de Cultura Económica: Buenos Aires.

Ricoeur, P. (2013). Escritos y conferencias 1. En torno al psicoanálisis. Editorial Trotta: Madrid. 